\title{
A New Formulation of Maximum Diversification Indexation Using Rao's Quadratic Entropy
}

Benoît Carmichael

Gilles Boevi Koumou

Kevin Moran

septembre / september 2015 


\section{Abstract}

This paper proposes a new formulation of the Maximum Diversification indexation strategy based on Rao's Quadratic Entropy (RQE). It clarifies the investment problem underlying the Most Diversified Portfolio (MDP) formed with this strategy, identifies the source of the MDP's out-of-sample performance, and suggests dimensions along which this performance can be improved. We show that these potential improvements are quantitatively important and are robust to portfolio turnover, portfolio risk, estimation window, and covariance matrix estimation.

Keywords : Rao's Quadratic Entropy, Portfolio Diversification, Maximum Diversification Indexation, Diversification Ratio, Most Diversified Portfolio

JEL Classification : G11

Carmichael: Département d'économique, Université Laval benoit.carmichael@ecn.ulaval.ca

Koumou: CIRPÉE et Département d'économique, Université Laval nettey-boevi-gilles-b.koumou.1@ulaval.ca Moran: CIRPÉE et Département d'économique, Université Laval kevin.moran@ecn.ulaval.ca

Early versions of this article were presented at the internal seminar of the Department of Economics (Université Laval), at the 48th Annual Conference of the Canadian Economics Association and at the 54 e Congrès de la SCSE. Support for this work has been provided by le Fonds Québécois de Recherche sur la Société et la Culture and le Centre Interuniversitaire sur le Risque, les Politiques Économiques et l'Emploi. 


\section{Introduction}

Risk-based indexing has recently become a popular alternative to traditional investing strategies based on the mean-variance model of Markowitz (1952). This approach determines portfolio allocations by relying only on the risk characteristics of assets, avoiding the need to provide estimates for expected returns and thus eliminating any loss associated with estimation errors (Chopra and Ziemba, 1993). ${ }^{1}$

One of the most popular risk-based investment strategy stems from Choueifaty and Coignard (2008)'s Maximum Diversification approach. It maximizes what the authors refer to as the diversification ratio (DR), and the portfolio that it generates is labeled the Most Diversified Portfolio (MDP). The MDP currently underlies the allocation choices of important managed funds worldwide: for example, it forms the basis of TOBAM's "Maximum Diversification index"which has attracted funds from global players like CALPERS. In addition, the FTSE group recently launched a new family of indices that follow this strategy. ${ }^{2}$

However, the source of the MDP's desirable properties has not been formally established and as a result, their validity and robustness to unforeseen shocks have often been questioned. For example, Lee (2011, p. 15-16) argues that the diversification ratio forming the basis of the strategy is a differential rather than an absolute diversification measure and as such cannot be used as a measure of portfolio diversification. ${ }^{3}$ In addition, he argues that no investment objective function is associated with the maximization of that ratio and that as a result, the investment problem underlying the MDP is undefined. Taliaferro (2012) also stresses the lack of clear investment objective underlying the $\mathrm{MDP}^{4}$ and suggests its success has been coincidental.

\footnotetext{
${ }^{1}$ Risk-based strategies, also known as beta smart indexing (Cazalet et al., 2014), include the minimum-variance and equal risk contribution portfolios, in addition to Choueifaty and Coignard (2008)'s Most Diversified Portfolio, the focus of the present paper.

${ }^{2}$ TOBAM (Think Out of the Box Asset Management) currently has 4.2 billion dollars under management (http: //www.tobam.fr/index.php). The funds following the MDP recently launched by the FTSE group include 5 regional and 3 single-country indices and are managed in collaboration with TOBAM.

${ }^{3} \mathrm{DR}$ is a differential diversification measure because it maximizes the difference between the volatility of the portfolio in an imaginary state (in which the correlation between all stocks is 1) and the volatility of the same portfolio in a real state.

4 "More curious is the MD methodology, which does not seek risk minimization, return maximization, Sharpe ratio maximization or the optimization of any other economically grounded measurement of investment performance. Instead, the MD methodology seeks portfolios with the greatest difference between pre- and post-formation risk, without regard for the risk or return profile of the resulting portfolio. Consequently, an MD portfolio only has desirable properties by accident. "(Taliaferro, 2012, p. 127)
} 
The present paper contributes to this debate in two important and related ways. First, we formally establishes the principles at play behind the Maximum Diversification approach. This is done by showing that MDP maximizes the ratio of portfolio RQE (Rao's Quadratic Entropy) to portfolio variance or, alternatively, minimizes portfolio variance subject to diversification constraint, where the diversification is measured by portfolio RQE. ${ }^{5}$ This formalization allows us to clarify the investment problem behind the MDP and helps identify the source of the MDP's strong out-of-sample performance relative to other diversified portfolios. Second, using this new formulation, we suggest directions along which the out-of-sample performances of the Maximum Diversification strategies can be improved and we show that these improvements are economically meaningful.

The remainder of this paper is organized as follows. Section 2 develops our new formulation of the Maximum Diversification (MD) indexation based on portfolio RQE and discusses its advantages over the standard formulation. Section 3 provides an empirical investigation that shows the improvements suggested by our reformulation are economically important. Section 4 verifies the robustness of our empirical findings and Section 5 concludes.

\section{A New Formulation of Maximum Diversification Indexation}

This section reformulates the problem of the Maximum Diversification indexation strategy in the context of Rao's Quadratic Entropy (RQE) and discusses two equivalent definitions of this formulation. Before proceeding, it is useful to recall the definitions of the diversification ratio (DR), which underlies the Maximum Diversification indexation strategy, as well as that of Rao's Quadratic Entropy of a portfolio.

\subsection{Maximum Diversification indexation}

Consider a world with $N$ risky assets. Let $\Sigma$ be the covariance matrix of returns, $\sigma=\left(\sigma_{1}, \cdots, \sigma_{N}\right)$ the vector of asset volatilities and $w=\left(w_{1}, \cdots, w_{N}\right)$ a long-only asset shares vector. Choueifaty and Coignard (2008)'s Maximum Diversification indexation strategy chooses $\omega$ so as to maximize the diversification ratio (DR)

$$
\max _{w \in \mathbf{W}} \operatorname{DR}(w)
$$

\footnotetext{
${ }^{5}$ Rao's Quadratic Entropy is a unifying measure of portfolio diversification analyzed by Carmichael et al. (2015)
} 
where $\mathbf{W}$ is the set of long-only portfolios and DR is the ratio of the weighted average of asset volatilities to portfolio volatility:

$$
\operatorname{DR}(w)=\frac{w^{\top} \sigma}{\sqrt{w^{\top} \Sigma w}}
$$

where $T$ is the transpose operator. An intuitive interpretation can be given to problem (1) in the special case where each asset's volatility is proportional to its expected premium, i.e.:

$$
\mathbb{E}\left(r_{i}\right)-r_{f}=\delta \sigma_{i}
$$

where $r_{i}$ is the return of asset $i, \sigma_{i}$ is its volatility, $r_{f}$ is the risk free rate return, $\delta>0$ and $\mathbb{E}(\cdot)$ is the expectation operator. In that case, the DR is proportional to the portfolio's Sharpe ratio and, as a result, problem (1) is therefore equivalent to maximizing the portfolio Sharpe ratio. However, note that relation (3) is not consistent with the CAPM although it can be justify theoretically by the results of Merton (1980) and Malkiel and Yexiao (2006). ${ }^{6}$ Other authors (Ang et al., 2006, 2009; Baker et al., 2011) report empirical evidence wherein high-volatility stocks have long underperformed low-volatility stocks, which casts serious doubts on the validity of (3). Furthermore, Lee (2011) stresses that (3) is not a no-arbitrage condition and, accordingly, the MDP investment problem is not well defined. Lee (2011) and Taliaferro (2012) argue that the MDP maximizes the distance between two volatility measures of the same portfolio. In an attempt to answer these concerns, Choueifaty et al. (2013) offer two alternative but these are not entirely satisfactory interpretations. ${ }^{7}$

\subsection{Rao's Quadratic Entropy}

Rao's Quadratic Entropy of a portfolio or portfolio Rao's Quadratic Entropy (RQE) is an approach for unifying portfolio diversification measures recently analyzed by Carmichael et al. (2015). This measure verifies ex-ante desirable properties for diversification metrics including duplication invariance. The authors also show that portfolio RQE is at the core of various existing measures of

\footnotetext{
${ }^{6}$ Martellini (2008) also reports empirical evidence which support this relation.

${ }^{7}$ For example, using empirical evidence, the authors claim that $\mathrm{DR}^{2}$ can be interpreted as the number of independent risk factors, or degrees of freedom, present in the portfolio, but this not the case generally and such interpretation can lead to counter-intuitive results. To illustrate, consider an universe of four assets and assume they all have the same volatility, with the correlation matrix defined as follows: $\rho_{12}=1, \rho_{3,4}=-1$ and $\rho_{i j}=0,(i, j) \neq(1,2)$ and $(3,4)$. One can show that $\operatorname{DR}^{2}\left(w^{M D}\right)=+\infty$, a counter-intuitive result.
} 
portfolio diversification, including the Gini-Simpson index and the diversification returns.

Let $w$ again denote a long only portfolio and define $D=\left(d_{i j}\right)_{i, j=1}^{N}$ a dissimilarity matrix between the various assets of the portfolio. The dissimilarity $d_{i j}$ measures the difference between assets $i$ and $j$ and can therefore be defined using a distance measure. One possible example of a dissimilarity matrix defines

$$
d_{i j}=1-\rho_{i j},
$$

where $\rho_{i j}$ measures the correlation between asset $i$ and $j$, so that asset $i$ and $j$ are considered more dissimilar the less they are correlated. Carmichael et al. (2015) define Rao's Quadratic Entropy as half of the mean difference between two randomly drawn (with replacement) assets from portfolio $w:$

$$
H_{D}(w)=\frac{1}{2} w^{\top} D w
$$

All thing equal, the higher $H_{D}(w)$ is, the more portfolio $w$ is diversified and a well-diversified portfolio can be obtained by maximizing (5). The specification of the dissimilarity matrix $D$ is left to portfolio managers, a flexibility that represents a major advantage of using portfolio RQE to measure diversification.

\subsection{Maximum Diversification meets Portfolio Rao's Quadratic Entropy}

We can now reconsider the diversification ratio in the context of portfolio RQE. In that context, note that the square of DR minus 1 gives

$$
\operatorname{DR}^{2}(w)-1=\frac{\left(w^{\top} \sigma\right)^{2}}{w^{\top} \Sigma w}-1
$$

Since (6) preserves the preference ordering on the set of long-only portfolios, the MDP can also be obtained by maximizing $\mathrm{DR}^{2}-1$. Notice further that (6) can also be written as (see Appendix for development)

$$
\operatorname{DR}^{2}(w)-1=\frac{w^{\top} \Gamma w}{w^{\top} \Sigma w},
$$


The numerator $w^{\top} \Gamma w$ is Rao's Quadratic Entropy (RQE) of portfolio $w$ with the dissimilarity matrix $\Gamma=\left(\gamma_{i j}\right)$ (portfolio $\mathrm{RQE}_{\Gamma}$ in short) and with typical element $\gamma_{i j}$

$$
\gamma_{i j}=\left(1-\rho_{i j}\right) \sigma_{i} \sigma_{j}
$$

In this formulation, $\gamma_{i j}$ measures the difference in terms of correlation and volatility between assets $i$ and $j$ and with (8): this difference is high when assets have high or moderate volatility, and low correlation. Portfolio RQE based on $\Gamma$ (portfolio $\mathrm{RQE}_{\Gamma}$ ) therefore selects moderate to high volatility assets with low correlation between them. Alternatively, diversification with $\mathrm{H}_{\Gamma}$ can be viewed as targeted towards the subset of moderate to high volatility assets.

As a result, one can interpret the ratio (7) as a diversification-risk trade-off, with diversification (the numerator) defined by portfolio $\mathrm{RQE}_{\Gamma}$ and risk (the denominator) defined by portfolio variance. Since $\mathrm{DR}^{2}-1$ and DR represent the same preference ordering on $\mathbf{W}$, we can deduce that the MDP investment problem can be rewritten as:

$$
w^{M D} \in \max _{w \in \mathbf{W}} \frac{w^{\top} \Gamma w}{w^{\top} \Sigma w}
$$

The formulation in (9) reveals that criticisms directed at the MDP may be unjustified: contrary to what Lee (2011) claims, MDP portfolios implicitly solve a well-specified investment problem that aims to maximize an absolute portfolio diversification measure normalized by the portfolio variance. As such, and contrary to arguments made in Taliaferro (2012), the MDP's desirable properties are probably not coincidental, but rather a result of this objective. For example, the MDP is duplication invariant because the minimum-variance (MVP) and maximum-RQE portfolios are both duplication invariant. ${ }^{8}$

Equation (9) also reveals that Maximum Diversification indexation (1) is a particular case of a more general portfolio allocation problem wherein

$$
w^{D} \in \max _{w \in \mathbf{W}} \frac{w^{\top} D w}{w^{\top} \Sigma w}
$$

\footnotetext{
${ }^{8}$ The maximum-RQE portfolio is the portfolio that maximizes RQE i.e $\mathrm{H}_{\Gamma}(w)$. It is also known under the name of RQE portfolios (see Carmichael et al., 2015). The duplication invariance of the MVP is proved in Choueifaty et al. (2013) and that of RQEP in Carmichael et al. (2015) for any dissimilarity matrix $D$.
} 
where $D$ is any dissimilarity matrix. This suggests that the out-of-sample performance of the MDP can potentially be improved using an alternative dissimilarity matrix. Section 3 provides an empirical illustration that shows this is indeed the case. In the remainder of this paper, we refer to problem (10) as the Rao's Quadratic Entropy ratio problem with dissimilarity matrix D, or more compactly $\mathrm{RQER}_{D}$. Its optimal portfolio is itself denoted $\mathrm{RQERP}_{D}$. This implies that the Maximum Diversification indexation strategy and $\mathrm{RQER}_{\Gamma}$ refer to the same problem.

\subsection{An Alternative formulation}

This section rewrites problem (9) as one of variance minimization subject to a diversification constraint measured by portfolio RQE. This alternative formulation of (9) facilitates the comparison between the MDP and other diversified portfolios such as the equal risk contribution (ERCP), the minimum-variance (MVP) and the 2-norm constrained minimum-variance (NC2P) (see Cazalet et al., 2014; DeMiguel et al., 2009a; Yanou, 2010) portfolios; in turn, this will serve: to better identify the source of the out-of-sample performance of the MDP.

Consider the following optimization problem

$$
\begin{gathered}
w^{D}(h) \in \min _{w \in \mathbf{W}} w^{\top} \Sigma w \\
\text { s.t } w^{\top} D w \geq h,
\end{gathered}
$$

where $h$ denotes the portfolio manager's minimum targeted level of diversification and $D$ is any dissimilarity matrix. It is straightforward to show that for dissimilarity matrix $\Gamma$, problems (9) and (11)-(12) are equivalent provided that $h=\mathrm{H}_{\Gamma}\left(w^{M D}\right)$ : the Maximum Diversification indexation strategy problem (Equation 1) is thus equivalent to (11)-(12) when $D=\Gamma$ and $h=\mathrm{H}_{\Gamma}\left(w^{M D}\right)$. Below, we refer to problem (11)-(12) as the constrained Rao's Quadratic Entropy problem or $\operatorname{RQEC}_{D, h}$, and denote its optimal portfolio $\operatorname{RQECP}_{D, h}$. We also denote $\mathrm{H}_{\Gamma}\left(w^{M D}\right)$ by $h_{\Gamma}$. This

implies that the Maximum Diversification indexation strategy, $\mathrm{RQER}_{\Gamma}$ and $\mathrm{RQEC}_{\Gamma, h_{\Gamma}}$ all refer to the same problem.

Formulating the Maximum Diversification strategy as $\mathrm{RQEC}_{\Gamma, h_{\Gamma}}$ has two advantages. The first is that it helps clarify the fact that, similarly to the ERCP and the NC2P, the MDP is the solution to 
a minimum-variance problem subject to a diversification constraint. This similitude is made clear when the different optimization problems underlying theses portfolios are compared:

$$
\begin{array}{ll}
\mathrm{NC} 2 \mathrm{P}: & w^{N C 2} \in \min _{w \in \mathbf{W}} w^{\top} \Sigma w \\
& \text { s.t } \sum_{i \neq j=1}^{N} w_{i} w_{j} \geq \nu \\
\mathrm{MVP}: & w^{M V} \in \min _{w \in \mathbf{W}} w^{\top} \Sigma w, \\
\mathrm{ERCP}: & w^{E R C} \in \min _{w \in \mathbf{W}} w^{\top} \Sigma w \\
& \text { s.t } \sum_{i=1}^{N} \frac{\mathbf{1}}{N} \ln \left(\frac{\mathbf{1} / N}{w_{i}}\right) \leq-\frac{\mathbf{1}}{N}(c+N \ln (N))
\end{array}
$$

Note that the left-hand sides of the diversification constraints (14) and (17) are respectively the portfolio Gini-Simpson index and the Kullback-Leibler divergence of the naive portfolio relative to the portfolio $w$.

Comparing problems (13)-(14), (15) and (16)-(17) to $\mathrm{RQEC}_{\Gamma, h_{\Gamma}}$, we notice that the difference lies in the presence of $\mathrm{RQE}_{\Gamma}$ in the diversification constraint (12). Therefore, the source of any differences in volatility, turnover, drawdown risk, concentration and Sharpe ratio between the MDP on the one hand and the NC2P, the MVP and the ERCP on the other originates from the portfolio $\mathrm{RQE}_{\Gamma}$ diversification constraint, which is characterized by the dissimilarity matrix $\Gamma$ and the minimum targeted level of diversification $h_{\Gamma} \cdot{ }^{9}$

It follows that if the diversification constraint is correctly specified, a significant portion of the out-of-sample performance of the MDP arises from the returns to diversification as measured by portfolio RQE. This result is consistent with Choueifaty and Coignard (2008) :

"Furthermore, if we continuously rebalance the Most-Diversified Portfolio, and because it is a market cap-independent methodology, the Most-Diversified Portfolio should get a significant part of the benefits from diversification returns when compared to a pure

\footnotetext{
${ }^{9}$ Generally, the MDP is less concentrated (in terms of weight), more volatile, has higher turnover and track-error (with benchmark the Capitalization-weighted), and a lower drawdown risk than the MVP. Choueifaty and Coignard (2008); Choueifaty et al. (2013) and Demey et al. (2010) find that the MDP is outperformed by the MVP, while Chow et al. (2011), Leote et al. (2012) and Clarke et al. (2013) report opposite. These authors also report that the MDP generally outperforms the ERCP, and it is most concentrated and less volatile.
} 
buy-and-hold strategy..."

When the diversification constraint is incorrect, one can use (11)-(12) to show that the MDP solves the modified minimum-variance problem

$$
w^{M D} \in \min _{w \in \mathbf{W}} w^{\top} \tilde{\Sigma} w
$$

where the true covariance matrix in (15) has been replaced by the matrix $\tilde{\Sigma}$ defined as

$$
\tilde{\Sigma}=\sigma^{\top}\left[\rho-\lambda\left(\iota \iota^{\top}-\rho\right)\right] \sigma
$$

with $\lambda$ the Lagrange multiplier associated to the constraint (12), $\rho$ the correlation matrix and $\iota$ the unit column vector. This shows, in the spirit of Ma and Jagannathan (2003), that the imposed RQE diversification constraint can be interpreted as a shrinking of the correlation matrix toward $-\iota \iota^{\top}$, which may help reduce potentially upward-biased estimates. This reduction in turn means that even when the diversification constraint is incorrectly specified, the MDP has the potential to produce better out-of-sample performances. This advantage depends on the trade-off between the reduction in sampling errors and the specification errors generated by the diversification constraint. The second advantage of the constrained $\mathrm{RQEC}_{\Gamma, h_{\Gamma}}$ formulation arises because it reveals that one can potentially improve the out-of-sample performance of the MDP, not only by replacing the dissimilarity matrix $\Gamma$ by a more suitable one, but also by choosing a more appropriate level of diversification $h$, different from $h_{\Gamma}$.

In short, our new formulation reveals that the superior out-of-sample performances of the MDP relative to the MVP, the ERCP and the $\mathrm{NC} 2 \mathrm{P}$ are due either to the returns to diversification when the diversification constraint that is employed is correct, or to the reduction of the upward-biased estimates in the correlation matrix when it is not. This leads us to the general conclusion that the funds under MD indexation management should not be considered systematically at risk. Moreover, when the diversification constraint is incorrect and the specification errors exceed the sample errors, our new formulation reveals that one can potentially improve the MDP's out-of-sample performance by changing the dissimilarity matrix $\Gamma$ and the minimum targeted level of diversification $h_{\Gamma}$ behind 
the MDP.

\section{Improving MDP out-of-sample performance}

As demonstrated above, the Maximum Diversification indexation investment strategy represents a special case of the more general unconstrained $\left(\mathrm{RQER}_{\mathrm{D}}, D=\Gamma\right)$ and constrained $\left(\mathrm{RQEC}_{\mathrm{D}, \mathrm{h}}, D=\right.$ $\Gamma$ and $h=h_{\Gamma}$ ) problems based on portfolio Rao's Quadratic Entropy. This implies that the approach underlying Maximum Diversification indexation could potentially be improved through the use of alternative specification for the dissimilarity matrix $D$ and the minimum targeted level of diversification $h$.

This section provides an empirical illustration that shows such improvements are indeed present and economically meaningful. To do so, we compare the simulated out-of-sample performances of five portfolios with those of the MDP. Each portfolio is obtained from problem (9) using different dissimilarity matrix $D$, or from problem (11)-(12), using different dissimilarity matrix $D$ and minimum targeted diversification level $h$.

\subsection{Methodology}

We consider three different procedures to construct the alternative portfolios. The first (Scenario I, top panel of Table 1) looks at the effect of changing the minimum level of diversification $h_{\Gamma}$ while keeping the dissimilarity matrix of the MDP $(\Gamma)$. Following DeMiguel et al. (2009a), we choose $h$ to maximize the portfolio return in the last period of the estimation window and the value of $h$ drawn from this procedure is denoted $h_{r}$ in Table 1 . This scenario is implemented using the constrained problem $\operatorname{RQEC}_{D, h}$.

The second procedure (Scenario II, middle panel of Table 1) looks at the effect of changing the dissimilarity matrix using the unconstrained problem $\mathrm{RQER}_{D}$. We consider three dissimilarity matrices other than the benchmark $\Gamma: \Phi, \Pi$ and $\Psi$. First, the matrix $\Phi$ assumes that assets are equi-dissimilar, so that the portfolio RQE based on $\Phi$ is equivalent to the Gini-Simpson index. In turn, the problem $\mathrm{RQER}_{\Phi}$ reproduces the $\mathrm{NC} 2$ problem when $\tau=h_{\Phi}$. Next, the matrix $\Pi$ is defined by replacing the volatility in $\Gamma$ by the upside-risk. Portfolio $\mathrm{RQE}_{\Pi}$ thus selects moderate to high upside-risk assets that are less correlated. Finally, the matrix $\Psi$ uses the ratio of upside to 
downside risk to replace the volatility in $\Gamma$; portfolio $\mathrm{RQE}_{\Psi}$ therefore selects assets with moderate to high ratios of upside to downside-risk which are less correlated. Portfolio $\mathrm{RQE}_{\Pi}$ and $\mathrm{RQE}_{\Psi}$ can also be interpreted as diversification among the subset of moderate to high upside risk assets, and on the subset of assets with moderate to high ratios of upside-risk to downside-risk i.e "targeted diversifications".

Finally, a third procedure (Scenario III, bottom panel of Table 1) analyzes change to dissimilarity matrix $\Gamma$ using the constrained problem $\operatorname{RQEC}_{D, h}$. The dissimilarity matrices considered remain $\Phi, \Pi$ and $\Psi$ and we choose $h$ following DeMiguel et al. (2009a) $\left(h=h_{r}\right)$. The difference between the scenarios II and III thus lays in the specification of the minimum targeted level of diversification $h$ : for scenario II, $h=H_{D}\left(w^{D}\right)$ is specified implicitly such the diversification per unity of risk is maximized, while in scenario III, $h$ is specified explicitly following DeMiguel et al. (2009a). Identifying the best specification for a given dissimilarity matrix $D$ requires comparing $\mathrm{RQECP}_{D, h_{r}}$ with RQERP ${ }_{D}$.

Our empirical work is based on Fama and French's twenty-five monthly portfolios returns sorted by size and book-to-market covering the period running from July 1963 to December $2013 .{ }^{10}$ Following DeMiguel et al. (2009a), we construct our portfolio returns using a rolling sample procedure with estimation window length $\tau=120$ and monthly rebalancing. The procedure generates a time series of $T-\tau-1$ monthly out-of-sample returns : $r_{t+1}^{j}=w_{t}^{j^{\top}} r_{t+1}, t=\tau, \ldots, T-1$, where $r_{t+1}$ is the vector of period $t+1$ asset returns and $w_{t}^{j}$ the portfolio weight vector at time $t$ for strategy $j$. Using this time series, we evaluate performance by reporting each portfolio's cumulative return $\left(\mathrm{CR}^{j}\right)$, its variance, $\left(\left(\sigma^{j}\right)^{2}\right)$ its Sharpe ratio $\left(\mathrm{SR}^{j}\right)$ and, finally, its turnover $\left(\mathrm{TRN}^{j}\right)$. More formally, these performances metrics are defined as follows:

$$
\begin{gathered}
\mathrm{CR}^{j}=\prod_{t=\tau}^{T-1}\left(1+w_{t}^{j^{\top}} r_{t+1}\right) \\
\left(\sigma^{j}\right)^{2}=\frac{1}{T-\tau-1} \sum_{t=\tau}^{T-1}\left(w_{t}^{j^{\top}} r_{t+1}-\mu_{j}\right)^{2} \text { with } \mu^{j}=\frac{1}{T-\tau} \sum_{t=\tau}^{T-1} w_{t}^{j^{\top}} r_{t+1} ;
\end{gathered}
$$

\footnotetext{
${ }^{10}$ The Dataset is available at http://mba.tuck.dartmouth.edu/pages/faculty/ken.french/data_library.html
} 
Table 1: List of Portfolios Considered

Dissimilarity matrix $(D) \quad$ Target Level of Diversification $(h)$ Portfolios

\section{Scenario I}

$\begin{array}{lll}\gamma_{i j}=\left(1-\rho_{i j}\right) \sigma_{i} \sigma_{j} & h_{\Gamma} & \operatorname{MDP}^{\dagger}\left(\operatorname{RQECP}_{\Gamma, h_{\Gamma}}\right) \\ \gamma_{i j}=\left(1-\rho_{i j}\right) \sigma_{i} \sigma_{j} & h_{r} & \operatorname{RQECP}_{\Gamma, h_{r}}\end{array}$

\section{Scenario II}

$\gamma_{i j}=\left(1-\rho_{i j}\right) \sigma_{i} \sigma_{j}$
$\phi_{i j}=1-\delta_{i j}$
$\pi_{i j}=\left(1-\rho_{i j}\right) \sigma_{i}^{+} \sigma_{j}^{+}$
$\psi_{i j}=\left(1-\rho_{i j}\right)\left(\frac{\sigma_{i}^{+}}{\sigma_{i}^{-}}\right)\left(\frac{\sigma_{j}^{+}}{\sigma_{j}^{-}}\right)$

Scenario III

$\gamma_{i j}=\left(1-\rho_{i j}\right) \sigma_{i} \sigma_{j}$

$\phi_{i j}=1-\delta_{i j}$

$\pi_{i j}=\left(1-\rho_{i j}\right) \sigma_{i}^{+} \sigma_{j}^{+}$

$\psi_{i j}=\left(1-\rho_{i j}\right)\left(\frac{\sigma_{i}^{+}}{\sigma_{i}^{-}}\right)\left(\frac{\sigma_{j}^{+}}{\sigma_{j}^{-}}\right)$

$\begin{array}{ll}- & \operatorname{MDP}^{\dagger}\left(\operatorname{RQERP}_{\Gamma}\right) \\ - & \operatorname{RQERP}_{\Phi} \\ - & \operatorname{RQERP}_{\Pi} \\ - & \operatorname{RQERP}_{\Psi}\end{array}$

$h_{r}$

$\mathrm{RQECP}_{\Gamma, h_{r}}^{\dagger}$ $\operatorname{RQECP}_{\Phi, h_{r}}$ $\mathrm{RQECP}_{\Pi, h_{r}}$ $\operatorname{RQECP}_{\Psi, h_{r}}$

Notes. This table lists the various portfolios we consider. The minimum targeted level of diversification $h_{r}$ is calibrated following DeMiguel et al. (2009a) to maximize the portfolio return in the last period within the estimation window. Note that $\sigma_{i}^{+}=\mathbb{E}\left(\max \left(0, r_{i}\right)^{3}\right)$ is the upside Risk, $\sigma_{i}^{-}=\mathbb{E}\left(\min \left(0, r_{i}\right)^{2}\right), \delta_{i j}$ is the Kronecker delta $\left(\delta_{i i}=1 ; \delta_{i j}=0 i \neq j\right)$ and the portfolio $\operatorname{RQECP}_{\Phi, h_{r}}$ is exactly the 2-norm-constrained minimum-variance portfolio investigated by DeMiguel et al. (2009a). Recall that MDP $\equiv \operatorname{RQECP}_{\Gamma, h_{\Gamma}} \equiv$ RQERP $_{\Gamma}$.

$\dagger$ : benchmark portfolio.

$$
\begin{gathered}
\mathrm{SR}^{j}=\frac{\mu^{j}}{\sigma^{j}} \\
\mathrm{TRN}^{j}=\frac{1}{T-\tau-1} \sum_{t=\tau}^{T-1} \sum_{i=1}^{N}\left(\left|w_{i, t+1}^{j}-w_{i, t^{+}}^{j}\right|\right) ;
\end{gathered}
$$

where $w_{i, t}^{j}$ is the portfolio weight in asset $i$ chosen at time $t$ under strategy $j, w_{i, t+}^{j}$ the portfolio weight before rebalancing at $t+1$, and $w_{i, t+1}^{j}$ the desired portfolio weight at $t+1$. We also evaluate the impact of turnover on performance metrics under non-zero transaction costs using the following portfolio return net of transaction cost:

$$
\underline{r}_{t+1}^{j}=\left(1-\kappa \sum_{i=1}^{N}\left|w_{i, t+1}-w_{i, t+}\right|\right)\left(1+w_{t}^{j^{\top}} r_{t+1}\right)-1
$$


where $\kappa$ is the transaction cost parameter (see DeMiguel et al., 2009b). Performances metrics (18)(20) are computed using portfolio returns net of proportional transaction costs (22) for different values of $\kappa$ ranging from 0.0001 to $0.005 .{ }^{11}$ We set the targeted return level of the upside-risk and downside-risk to zero, and their risk attitudes parameter equal to 3 and 2 respectively (see Notes of Table 1). To test the out-of-sample variance and Sharpe ratio differences between two given portfolios, we use the studentized circular block bootstrap proposed in Ledoit and Wolf (2011) and Ledoit and Wolf (2008) respectively. ${ }^{12}$.

\subsection{Results}

\subsubsection{Scenario I}

Figure 1 depicts the out-of-sample cumulative returns of $\mathrm{MDP}$ and $\mathrm{RQECP}_{\Gamma, h_{r}}$. It shows that $\operatorname{RQECP}_{\Gamma, h_{r}}$ is never dominated by the MDP. One dollar invested in $\operatorname{RQECP}_{\Gamma, h_{r}}$ in July 1973 yields 190 dollars in December 2013, while the same investment in MDP yields 81 dollars.

Table 2 compares the performance of both portfolios in term of variance, Sharpe ratio and turnover. It reveals that the MDP has essentially the same variance as $\operatorname{RQECP}_{\Gamma, h_{r}}$, but significantly lower Sharpe ratio. These evidences lead us to conclude that the implicit target level of diversification of the $\operatorname{MDP}\left(h_{\Gamma}\right)$ may not always be "optimal ".

Table 2 also shows that $\mathrm{RQECP}_{\Gamma, h_{r}}$ implies a significant more active management policy, since turnover is higher. Figure 2 evaluates the impact of this higher turnover for our performance metrics when transaction costs are non zero. Only results for Sharpe ratio and cumulative returns at the end-of-period are reported, but full results are available. The figure shows that $\mathrm{RQECP}_{\Gamma, h_{r}}$ always outperforms MDP for investors facing small to moderate transaction costs, like moderate and large funds.

\footnotetext{
${ }^{11}$ Balduzzi and Lynch (1999, pp. 63) argue that small investors probably face a proportional transaction cost closer to the 0.005 , while large investors likely face costs greater than the 0.0001 .

${ }^{12}$ We set the block length equal to 5 and iterations equal to 1000 to compute the two-sided p-value. The null hypothesis is : $H_{0}: 2 \log \left(\hat{\sigma}^{i}\right)-2 \log \left(\hat{\sigma}^{j}\right)=0$ for the variance test, and $H_{0}: \hat{\mu}^{i} / \hat{\sigma}^{i}-\hat{\mu}^{j} / \hat{\sigma}^{j}=0$ for the Sharpe ratio test. The code is available at http://www.econ.uzh.ch/en/faculty/wolf/publications.
} 
Figure 1: Scenario I: Portfolios Cumulative Returns

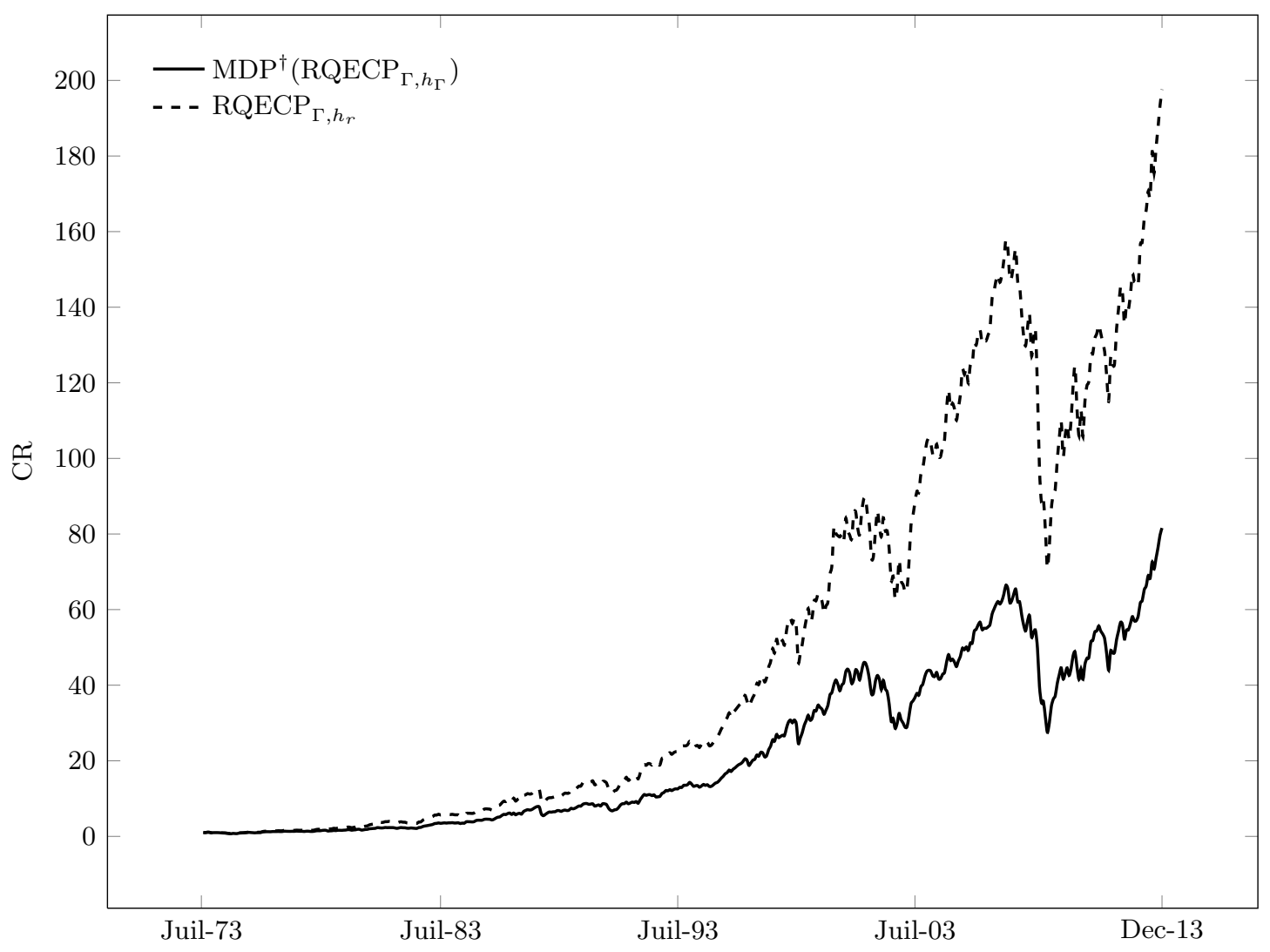

$\dagger$ : benchmark portfolio.

Table 2: Scenario I: Portfolios Performances

\begin{tabular}{lccc}
\hline Portfolios & $\sigma^{2}$ & SR & TRN \\
\hline $\operatorname{MDP}^{\dagger}\left(\mathrm{RQECP}_{\Gamma, h_{\Gamma}}\right)$ & 0.00226 & 0.21537 & 0.06337 \\
$\operatorname{RQECP}_{\Gamma, h_{r}}$ & 0.00227 & $0.25329^{*}$ & 0.75830 \\
\hline
\end{tabular}

$\dagger$ : benchmark portfolio.

Significance Level: $*=\mathrm{p}$-value $\leq 5 \%$ means that performances of RQECP $_{\Gamma, h_{r}}$ is significantly different from that of the benchmark. 
Figure 2: Scenario I: Performance metrics depending on transaction costs parameters $\kappa$

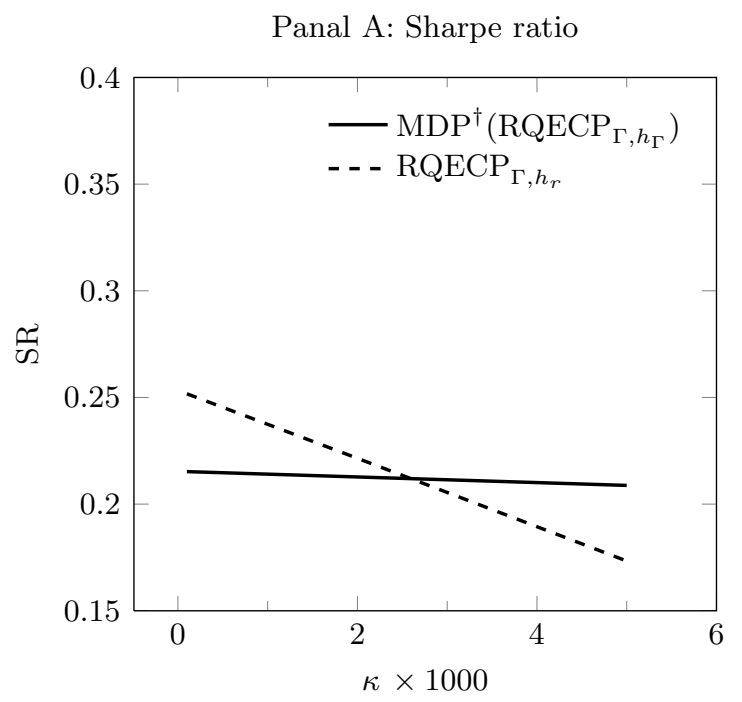

Panal B: Cumulative return (end-of-period)

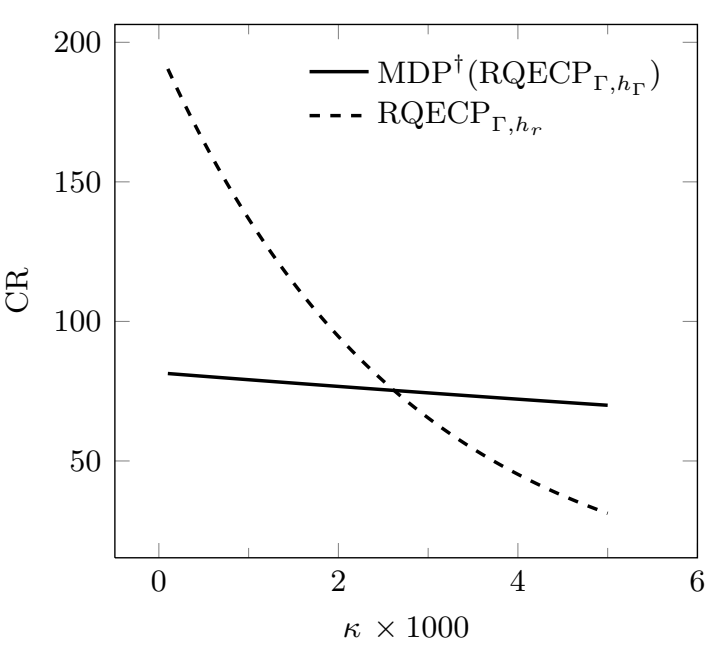

$\dagger$ : benchmark portfolio.

\subsubsection{Scenario II}

Figure 3 depicts the cumulative returns of $\mathrm{MDP}, \mathrm{RQERP}_{\Pi}, \mathrm{RQERP}_{\Psi}$ and $\mathrm{RQERP}_{\Phi}$. It shows that $\operatorname{RQERP}_{\Phi}$ and $\operatorname{RQERP}_{\Psi}$ are never dominated by MDP, while the performance of $\operatorname{RQERP}_{\Pi}$

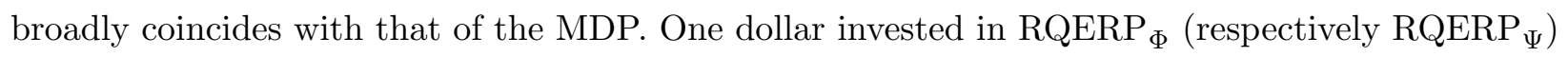
in July 1973 yields, in December 2013, 1.6 (respectively 1.7) times the cumulative return of one dollar invested in the MDP.

Table 3 reports the portfolios' performances in term of variance, Sharpe ratio and turnover. It shows that $\mathrm{RQERP}_{\Pi}$ offers essentially the same performance in term of Sharpe ratio as the MDP, but with a significantly higher variance. $\mathrm{RQERP}_{\Psi}$ offers essentially the same performance in term of variance as the MDP, but with a significantly higher Sharpe ratio. $R_{Q E R P}$ has significantly lower variance and higher Sharpe ratio than the MDP. Again, these evidences suggest that the implicit dissimilarity matrix of MDP is not always "optimal".

In addition, Table 3 also shows that $\operatorname{RQERP}_{\Phi}$ and $\mathrm{RQERP}_{\Psi}$ have respectively lower and higher turnover than the MDP. We evaluate the impact of the higher turnover of $\operatorname{RQERP}_{\Psi}$ when transaction costs are present. Results (Figure 4) show that $\mathrm{RQERP}_{\Psi}$ always outperforms the MDP.This means that the presence of non-zero transaction costs is not sufficient to overturn our results. 
Figure 3: Scenario II: Portfolios Cumulative Returns

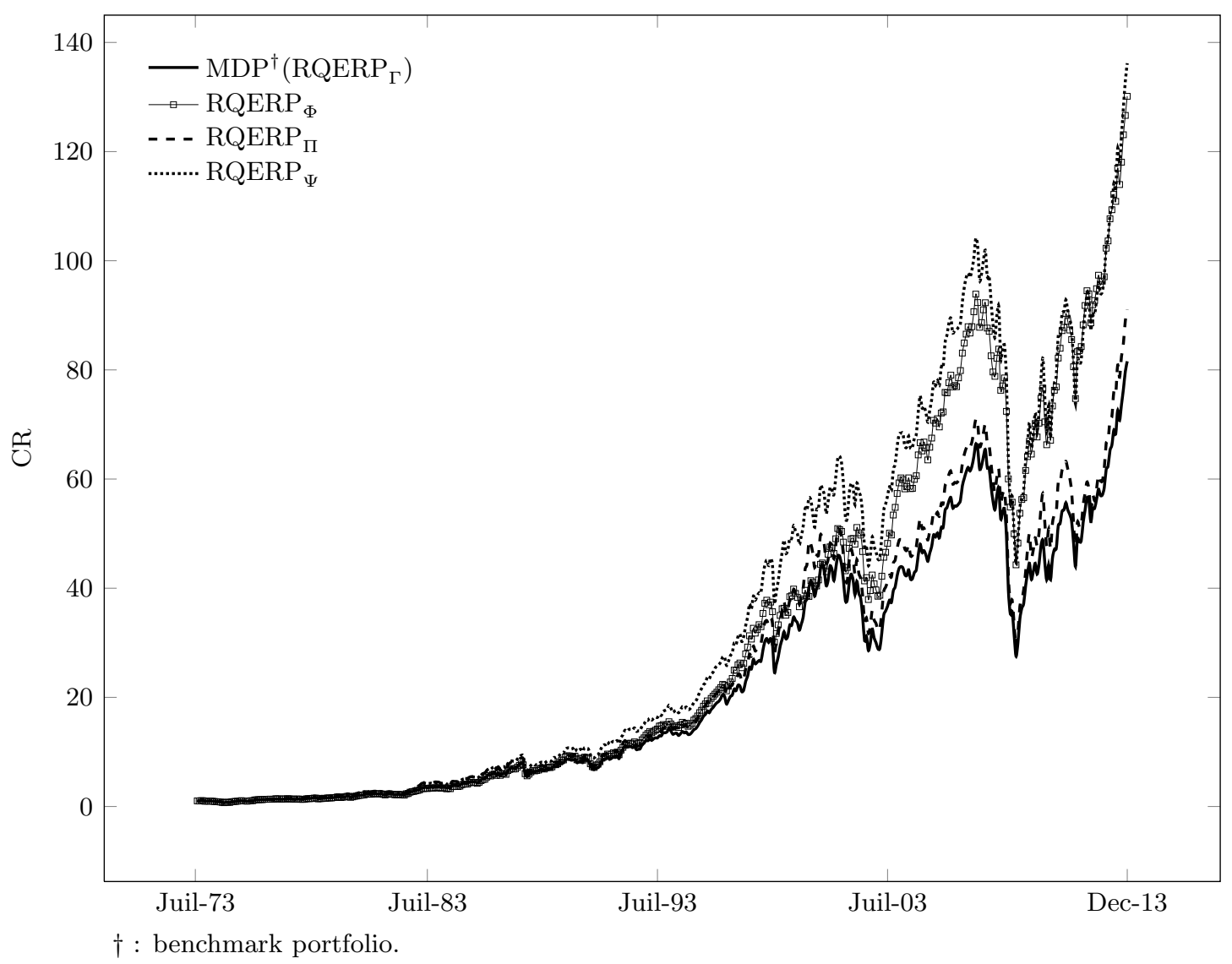

Table 3: Scenario II: Portfolios Performances

\begin{tabular}{lccc}
\hline Portfolios & $\sigma^{2}$ & SR & TRN \\
\hline $\operatorname{MDP}^{\dagger}\left(\mathrm{RQERP}_{\Gamma}\right)$ & 0.00226 & 0.21537 & 0.06337 \\
RQERP $_{\Phi}$ & $0.00191^{*}$ & $0.25194^{*}$ & 0.03992 \\
RQERP $_{\Pi}$ & $0.00251^{*}$ & 0.21136 & 0.08780 \\
$\operatorname{RQERP}_{\Psi}$ & 0.00215 & $0.24239^{*}$ & 0.09046 \\
\hline
\end{tabular}

$\dagger$ : benchmark portfolio.

Significance Level: $*=$ p-value $\leq 5 \%$ means that performances of $\operatorname{RQECP}_{\Phi}, \mathrm{RQECP}_{\Pi}$ and $\mathrm{RQECP}_{\Psi}$ is significantly different from that of the benchmark. 
Figure 4: Scenario II: Performance metrics depending on transaction costs parameters $\kappa$
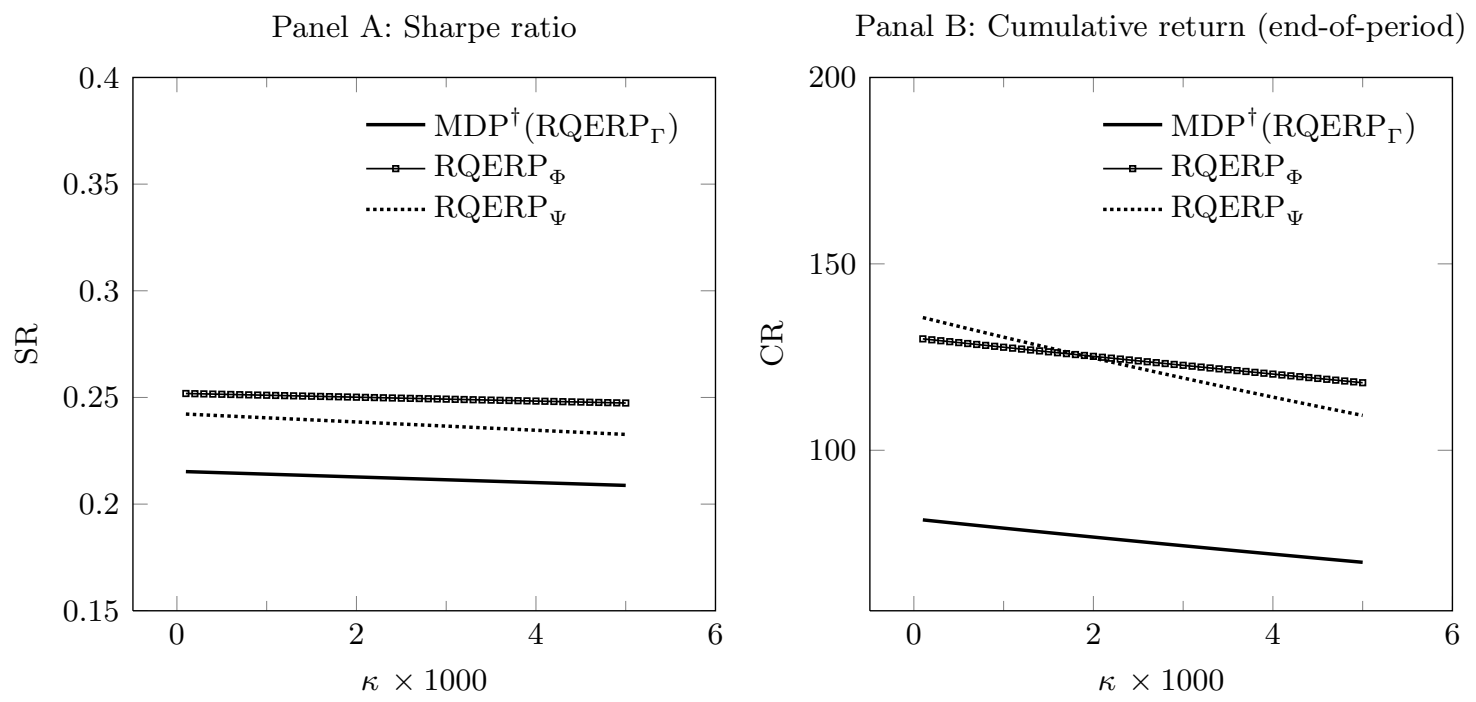

$\dagger$ : benchmark portfolio.

\subsubsection{Scenario III}

Figure 5 depicts the cumulative returns of $\mathrm{RQECP}_{\Gamma, h_{r}}, \mathrm{RQECP}_{\Phi, h_{r}}, \mathrm{RQECP}_{\Pi, h_{r}}$ and $\mathrm{RQECP}_{\Psi, h_{r}}$. It shows that $\mathrm{RQECP}_{\Gamma, h_{r}}$ is dominated by $\mathrm{RQECP}_{\Phi, h_{r}}, \mathrm{RQECP}_{\Pi, h_{r}}$ and $\mathrm{RQECP}_{\Psi, h_{r}}$ : One dollar invested in these three strategies in July 1973 yields $254\left(\mathrm{RQECP}_{\Phi, h_{r}}\right), 312$ dollars $\left(\mathrm{RQECP}_{\Pi, h_{r}}\right)$ and 225 dollars $\left(\operatorname{RQECP}_{\Psi, h_{r}}\right)$ in December 2013. By contrast, one dollars invests in $\mathrm{RQECP}_{\Gamma, h_{r}}$ at the same period delivers 197 dollars.

Table 4 shows that portfolio $\mathrm{RQECP}_{\Gamma, h_{r}}$ has a significantly higher variance than $\mathrm{RQECP}_{\Phi, h_{r}}$ and $\operatorname{RQECP}_{\Psi, h_{r}}$ but a lower one than $\operatorname{RQECP}_{\Pi, h_{r}}$. In terms of Sharpe ratio, $\operatorname{RQECP}_{\Gamma, h_{r}}$ is slightly dominated by $\operatorname{RQECP}_{\Phi, h_{r}}, \operatorname{RQECP}_{\Pi, h_{r}}$ and $\operatorname{RQECP}_{\Psi, h_{r}}$, but the difference are not significant. Again these lead us to conclude that the implicit dissimilarity matrix of MDP is not always "optimal".

Table 4 also shows that $\operatorname{RQECP}_{\Psi, h_{r}}$ has lower turnover than $\operatorname{RQECP}_{\Gamma, h_{r}}$, while $\operatorname{RQECP}_{\Phi, h_{r}}$ and $\mathrm{RQECP}_{\Pi, h_{r}}$ have higher turnover. We evaluate the impact of the higher turnover when transaction costs are present in Figure 6, which shows that $\operatorname{RQECP}_{\Phi, h_{r}}, \operatorname{RQECP}_{\Pi, h_{r}}$ and $\operatorname{RQECP}_{\Psi, h_{r}}$ always outperform RQECP $\operatorname{R,h}_{r}$. 
Figure 5: Scenario III: Portfolios Cumulative Returns

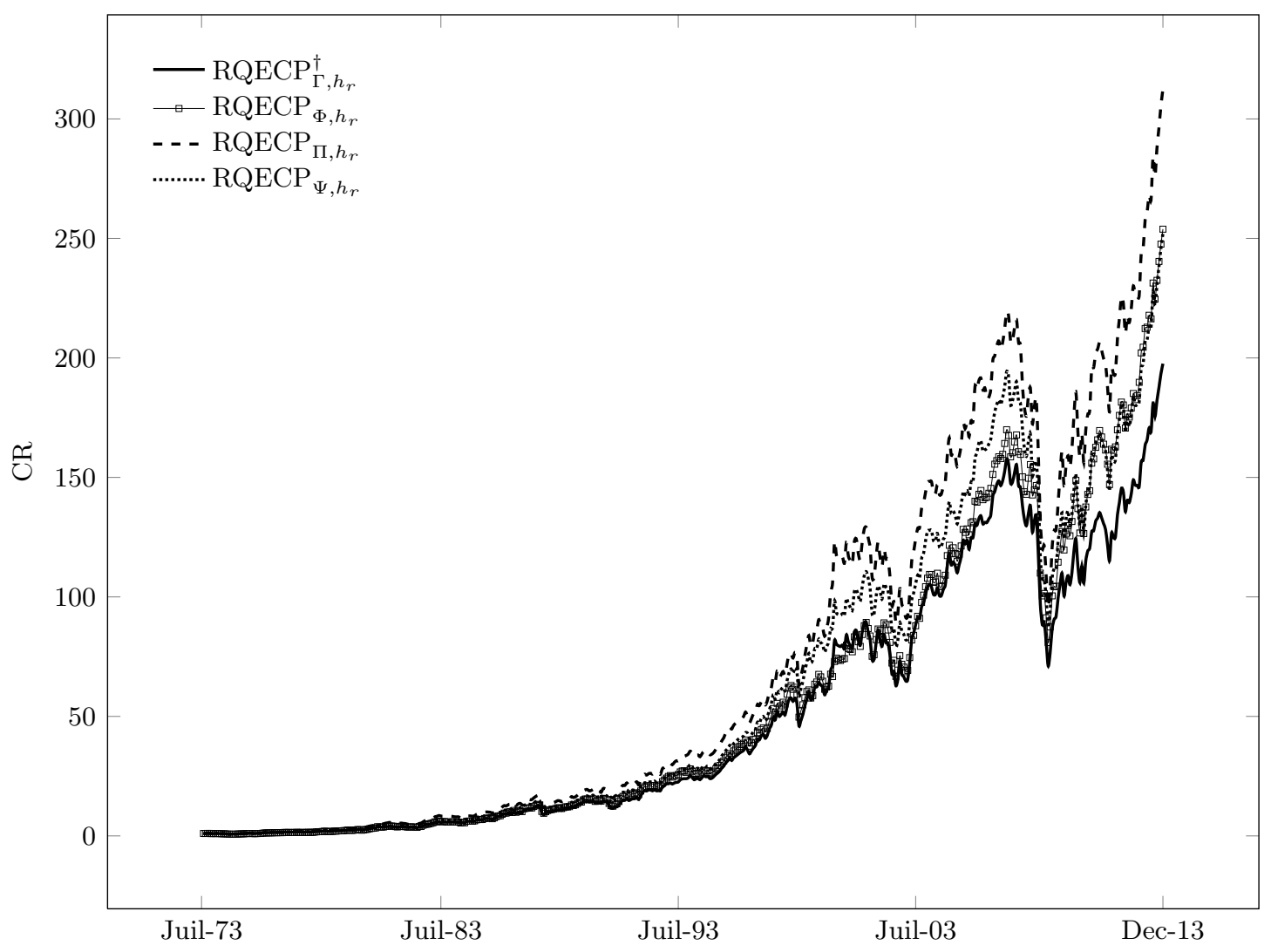

$\dagger$ : benchmark portfolio.

Table 4: Scenario III: Portfolios Performances

\begin{tabular}{lccc}
\hline Portfolios & $\sigma^{2}$ & SR & TRN \\
\hline RQECP $_{\Gamma, h_{r}}^{\dagger}$ & 0.00227 & 0.25329 & 0.75830 \\
RQECP $_{\Phi, h_{r}}$ & $0.00211^{*}$ & 0.27229 & 0.80755 \\
RQECP $_{\Pi, h_{r}}$ & $0.00240^{*}$ & 0.26724 & 0.89158 \\
RQECP $_{\Psi, h_{r}}$ & $0.00209^{*}$ & 0.27303 & 0.72992 \\
\hline
\end{tabular}

$\dagger$ : benchmark portfolio.

Significance Level: $*=\mathrm{p}$-value $\leq 5 \%$ means that performances of $\operatorname{RQECP}_{\Phi, h_{r}}, \operatorname{RQECP}_{\Pi, h_{r}}$, and $\operatorname{RQECP}_{\Psi, h_{r}}$ is significantly different from that of the benchmark. 
Figure 6: Scenario III: Performance metrics depending on transaction costs parameters $\kappa$
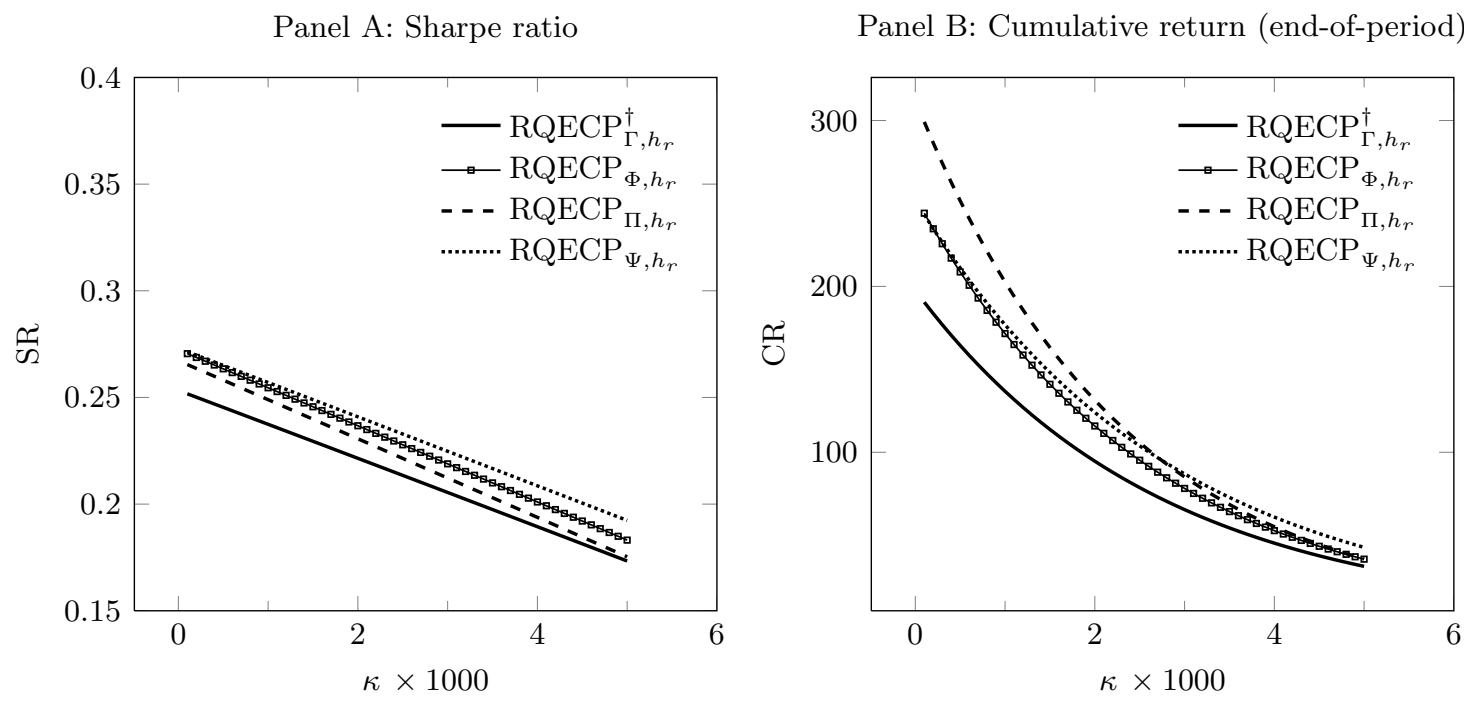

$\dagger$ : benchmark portfolio.

In short, while the Maximum Diversification indexation at the basis of the MDP is equivalent to the problems $\mathrm{RQER}_{\Gamma}$ and $\mathrm{RQEC}_{\Gamma, h_{\Gamma}}$, our results show that one can significantly improve the out-of-sample performance of this general strategy using different dissimilarity matrices and/or by changing the minimum targeted level of diversification. Note that the potential improvement are particularly important in terms of Sharpe ratio when asset excess returns are not perfectly proportional to its volatility, and low otherwise. The potential improvement can also be important in terms of cumulative returns when asset returns are not normally distributed.

\section{Robustness checks}

We check the robustness of our empirical findings by considering alternative portfolios risk, estimation window, and covariance matrix estimation.

\subsection{Portfolio risk}

To check robustness to portfolio risk, which we equate to portfolio variance or volatility, we consider alternative portfolio risk measures such as : semivariance (SV), Value at risk (VaR), Conditional value at risk or Expected Shortfall $(\mathrm{CVaR})$ and Maximum Drawdown (MDD). These risk measures 
are defined as follows:

$$
\begin{gathered}
\mathrm{SV}^{j}(w)=\frac{1}{T} \sum_{t=\tau}^{T}\left[\min \left(0, r_{t+1}^{j}-\mu^{j}\right)\right]^{2} \\
\operatorname{VaR}_{\alpha}^{j}(w)=-q_{\alpha}^{j-}(w) \\
\operatorname{CVaR}_{\alpha}^{j}(w)=\frac{1}{\alpha} \int_{0}^{\alpha} \operatorname{VaR}_{u}^{j}(w) d u \\
\operatorname{MDD}^{j}=\max _{t_{1} \in(\tau, T-1)}\left[\max _{t_{2} \in\left(\tau, t_{1}\right)}\left(r_{t_{1}+1}^{j}-r_{t_{2}+1}^{j}\right)\right]
\end{gathered}
$$

where $q_{\alpha}^{j-}(w)=\inf \left\{x \mid P\left(r^{j}(w) \leq x\right) \geq \alpha\right\}$ is the lower quantiles of $r^{j}$ of order $\alpha \in(0,1)$.

Table 5: Portfolios risk

\begin{tabular}{llcccc}
\hline & Portfolios & SV & VaR & CVaR & MDD \\
\hline \multirow{2}{*}{ Scenario I } & $\operatorname{MDP}^{\dagger}\left(\operatorname{RQECP}_{\Gamma, h_{\Gamma}}\right)$ & 0.00130 & -0.07151 & -0.10821 & 0.58557 \\
& $\operatorname{RQECP}_{\Gamma, h_{r}}$ & 0.00122 & -0.06478 & -0.10116 & 0.54941 \\
\hline \multirow{3}{*}{ Scenario II } & $\operatorname{MDP}^{\dagger}\left(\mathrm{RQERP}_{\Gamma}\right)$ & 0.00130 & -0.07151 & -0.10821 & 0.58557 \\
& $\operatorname{RQERP}_{\Phi}$ & 0.00108 & -0.06039 & -0.09806 & 0.52827 \\
& $\operatorname{RQERP}_{\Pi}$ & 0.00144 & -0.07789 & -0.11410 & 0.58808 \\
& $\operatorname{RQERP}_{\Psi}$ & 0.00124 & -0.06913 & -0.10538 & 0.57478 \\
\hline \multirow{3}{*}{ Scenario III } & $\operatorname{RQECP}_{\Gamma, h_{r}}^{\dagger}$ & 0.00122 & -0.06478 & -0.10116 & 0.54941 \\
& $\operatorname{RQECP}_{\Phi, h_{r}}$ & 0.00116 & -0.06334 & -0.09837 & 0.52396 \\
& $\operatorname{RQECP}_{\Pi, h_{r}}$ & 0.00128 & -0.06231 & -0.10225 & 0.56233 \\
& $\operatorname{RQECP}_{\Psi, h_{r}}$ & 0.00115 & -0.06080 & -0.09786 & 0.55067 \\
\hline
\end{tabular}

$\dagger$ : benchmark portfolio.

Table 5 reports the value of these different risk measures for our set of considered portfolios. In Scenario I, we can observe that $\mathrm{RQECP}_{\Gamma, h_{r}}$ dominates MDP for all risk measures. In Scenario II, RQERP ${ }_{\Pi}$ is dominated by MDP, which is itself dominated by $\operatorname{RQERP}_{\Phi}$ and $\mathrm{RQERP}_{\Psi}$ for all risk measures. In Scenario III, $\mathrm{RQECP}_{\Phi, h_{r}}$ dominates MDP for all risk measures. $\mathrm{RQECP}_{\Psi, h_{r}}$ dominates MDP for all risk measures, except for MDD. $\mathrm{RQECP}_{\Pi, h_{r}}$ dominates MDP only for VaR. As a result, our findings remain robust in terms of portfolio risk. 


\subsection{Estimation window}

Our empirical analysis assumes an estimation window of 120 months. To check whether our results are driven by this assumption, we consider alternative window lengths of 60 and 240 months. We report the resulting Sharpe ratios in Table 6. As we can observe, for $\tau=240$ and $\tau=$ 60 , the dissimilarity matrix behind the $\operatorname{MDP}\left(h_{\Gamma}\right)$ is "sub-optimal ", but the minimum level of diversification $\Gamma$ seems "optimal". In short, our findings are mostly robust for $\tau=240$ and $\tau=60$.

Table 6: Sharpe ratio

\begin{tabular}{|c|c|c|c|c|}
\hline & \multirow[b]{2}{*}{ Portfolios } & \multicolumn{2}{|c|}{ Rolling Window } & \multirow{2}{*}{$\begin{array}{c}\text { Robustness }(\Sigma) \\
\tau=120\end{array}$} \\
\hline & & $\tau=60$ & $\tau=240$ & \\
\hline \multirow{2}{*}{ Scenario I } & $\operatorname{MDP}^{\dagger}\left(\operatorname{RQECP}_{\Gamma, h_{\Gamma}}\right)$ & 0.17171 & 0.20072 & 0.21859 \\
\hline & $\mathrm{RQECP}_{\Gamma, h_{r}}$ & 0.20247 & 0.22940 & $0.25765^{*}$ \\
\hline \multirow{4}{*}{ Scenario II } & $\mathrm{MDP}^{\dagger}\left(\mathrm{RQERP}_{\Gamma}\right)$ & 0.17171 & 0.20072 & 0.21859 \\
\hline & $\operatorname{RQERP}_{\Phi}$ & $0.22667^{*}$ & $0.25425^{*}$ & $0.25164^{*}$ \\
\hline & $\mathrm{RQERP}_{\Pi}$ & 0.14337 & 0.17806 & 0.21323 \\
\hline & $\operatorname{RQERP}_{\Psi}$ & 0.21550 & 0.22132 & $0.24453^{*}$ \\
\hline \multirow{4}{*}{ Scenario III } & $\mathrm{RQECP}_{\Gamma, h_{r}}^{\dagger}$ & 0.20247 & 0.22940 & 0.25765 \\
\hline & $\mathrm{RQECP}_{\Phi, h_{r}}$ & 0.22551 & $0.25210^{*}$ & 0.27229 \\
\hline & $\mathrm{RQECP}_{\Pi, h_{r}}$ & 0.18535 & 0.17961 & 0.27001 \\
\hline & $\operatorname{RQECP}_{\Psi, h_{r}}$ & 0.22410 & 0.21184 & 0.27387 \\
\hline
\end{tabular}

Significance Level: $*=\mathrm{p}$-value $\leq 5 \%$.

$\dagger$ : benchmark portfolio.

\subsection{Covariance matrix estimation}

Our results are based on the sample covariance matrix. To check whether an alternative specification would be more appropriate, we consider a covariance matrix using Ledoit and Wolf (2003)'s shrinkage estimation, where the shrinkage target is obtained from a one-factor model where the factor is equal to the cross-sectional average of all the random variables. ${ }^{13}$ As we can observe from Table 6, our findings are unchanged by this. In Scenario I, the MDP still has the lower out-ofsample Sharpe ratio. In Scenario II, we can find a dissimilarity matrix that out-performs $\Gamma$ ( $\Phi$ and $\Psi)$. In short, our finding are robust to covariance matrix estimation.

\footnotetext{
${ }^{13}$ Computations are made with the code available at https://r-forge.r-project.org/scm/viewvc.php/pkg/ ExpectedReturns/man/?root=expectedreturns\&pathrev=2.
} 


\section{Conclusion}

In this paper, we have proposed a new formulation for the Maximum Diversification indexation strategy using portfolio Rao's Quadratic Entropy (RQE). We show that the Most Diversified Portfolio (MDP) maximizes the ratio of portfolio RQE to portfolio variance or, said differently, minimizes portfolio variance subject to a diversification constraint. This new formulation reveals that the Maximum Diversification strategy solves a well-defined investment objective.

Moreover, our new formulation also suggests two new directions to improve the out-of-sample performances of the approach: we show that the MDP's out-of-sample performances can be significantly improved by changing its dissimilarity matrix and its minimum level of diversification. Our robustness analysis has shown that these results are not dependent on risk measure, turnover, estimation window and covariance matrix estimation. The portfolio allocation problem (11)-(12) constitutes therefore a very credible alternative to the Maximum Diversification indexation strategy, particularly when asset excess returns are not perfectly proportional to its volatility or when asset returns are not normally distributed. Additional research could compare the out-of-sample performance of the optimal portfolio of problem (11)-(12) across different empirical datasets for various dissimilarity matrices and minimum level of diversification specifications. 


\section{References}

Ang, A., R. J. Hodrick, Y. XIng, And X. Zhang (2006). "The Cross-Section of Volatility and Expected Returns," Journal of Finance, 61, 259-299.

(2009). "High idiosyncratic volatility and low returns: International and further U.S. evidence," Journal of Financial Economics, 91, 1-23.

Baker, M., B. Bradley, and J. Wurgler (2011). "Benchmarks as Limits to Arbitrage: Understanding the Low-Volatility Anomaly," Financial Analysts Journal, 67, 1-15.

Baker, N. L. And R. A. Haugen (2012). "Low Risk Stocks Outperform within All Observable Markets of the World," Tech. rep.

BAlduzzi, P. AND A. W. LynCh (1999). "Transaction costs and predictability: some utility cost calculations," Journal of Financial Economics, 52, 47 - 78.

Carmichael, B., G. B. Koumou, and K. Moran (2015). "Unifying Portfolio Diversification Measures Using Rao's Quadratic Entropy," Cirano working papers, CIRANO.

Cazalet, Z., P. Grison, and T. Roncalli (2014). "The Smart Beta Indexing Puzzle," The Journal of Index Investing, 5, 97-119.

Chopra, V. K. And W. T. Ziemba (1993). "The Effect of Errors in Means, Variances, and Covariances on Optimal Portfolio Choice," Journal of Portfolio Management, 19, 6-11.

Choueifaty, Y. And Y. Coignard (2008). "Toward Maximum Diversification," Journal of Portfolio Management, 35, 40-51.

Choueifaty, Y., T. Froidure, And J. Reynier (2013). "Properties of the most diversified portfolio," Journal of Investment Strategies, 2, 49-70.

Chow, T.-M., J. Hsu, V. Kalesnik, And B. Little (2011). "A Survey of Alternative Equity Index Strategies," Financial Analysts Journal, 17, 37-57.

Clarke, R., H. De Silva, and S. Thorley (2013). "Risk Parity, Maximum Diversification, and Minimum Variance: An Analytic Perspective," Journal of Portfolio Management, 29, 39-53. 
Demey, P., S. Maillard, And T. Roncalli (2010). "Risk-Based Indexation," White Paper 1, Lyxor.

DeMiguel, V., L. Garlappi, F. J. Nogales, and R. Uppal (2009a). "A Generalized Approach to Portfolio Optimization: Improving Performance by Constraining Portfolio Norms," Management Science, 55, 798-812.

DeMiguel, V., L. Garlappi, and R. Uppal (2009b). "Optimal Versus Naive Diversification : How Inefficient is the 1/N Portfolio Strategy?" The Review of Financial Studies, 22, 1915-1953.

Haugen, R. A. And N. L. BAKer (1991). "The efficient market inefficiency of capitalizationweighted stock portfolios," Journal of Portfolio Management, 17, 35-40.

LEDOIT, O. AND M. Wolf (2003). "Improved estimation of the covariance matrix of stock returns with an application to portfolio selection," Journal of Empirical Finance, 10, 603-621.

(2008). "Robust performance hypothesis testing with the Sharpe ratio," Journal of Empirical Finance, 15, 850-859.

— (2011). "Robust Performances Hypothesis Testing With the Variance," Wilmott, 2011, 8689.

LeE, W. (2011). "Risk-Based Asset Allocation: A New Answer to an Old Question? ," Journal Of Portfolio Management, 37, 11-28.

Leote, R., X. Lu, And P. Moulin (2012). "Demystifying Equity Risk-Based Strategies: A Simple Alpha plus Beta Description," Journal of Portfolio Management, 38, 56-70.

Ma, T. and R. Jagannathan (2003). "Risk Reduction in Large Portfolios: Why Imposing the Wrong Constraints Helps," Journal of Finance, 58, 57-72.

Maillard, S., T. Roncalli, and J. Teïletche (2010). "The Properties of Equally Weighted Risk Contribution Portfolios," Journal of Portfolio Management, 36, 60-70.

Malkiel, B. G. And X. Yexiao (2006). "Idiosyncratic Risk and Security Returns," Tech. rep., https://www.utdallas.edu/ yexiaoxu/IVOT_H.PDF. 
Markowitz, H. (1952). "Portfolio Selection," The Journal of Finance, 7, 77-91.

Martellini, L. (2008). "Toward the Design of Better Equity Benchmarks: Rehabilitating the Tangency Portfolio from Modern Portfolio Theory," Journal of Portfolio Management, 34, p441.

Merton, R. C. (1980). "On Estimating the Expected Return on the Market: An Exploratory Investigation," Journal of Financial Economics, 8, 323-361.

— (1987). "A Simple Model of Capital Market Equilibrium with Incomplete Information," Journal of Finance, 42, 41-81.

Meucci, A. (2009). "Managing Diversification," Risk, 22, 74-79.

TAliaferro, R. (2012). "Understanding risk-based portfolios," The Journal of Investment Strategies, 1, 119-131.

Yanou, G. (2010). "Mean-Variance Framework and Diversification Objective: Theoretical and Empirical Implications," Tech. rep., University of Paris-1, http://ssrn. com/abstract=1722285. 


\section{Appendix Development of $\mathrm{DR}^{2}-1$}

The development of $\left(w^{\top} \sigma\right)^{2}$ and $w^{\top} \Sigma w$ are

$$
\left(w^{\top} \sigma\right)^{2}=\sum_{i=1}^{N} w_{i}^{2} \sigma_{i}^{2}+\sum_{i, j=1}^{N} w_{i} w_{j} \sigma_{i} \sigma_{j} \quad \text { and } \quad w^{\top} \Sigma w=\sum_{i=1}^{N} w_{i}^{2} \sigma_{i}^{2}+\sum_{i, j=1}^{N} w_{i} w_{j} \rho_{i j} \sigma_{i} \sigma_{j} .
$$

Therefore,

$$
\mathrm{DR}^{2}(w)-1=\frac{\sum_{i, j=1}^{N}\left(1-\rho_{i j}\right) \sigma_{i} \sigma_{j} w_{i} w_{j}}{w^{\top} \Sigma w}=\frac{w^{\top} \Gamma w}{w^{\top} \Sigma w}
$$

where $\Gamma=\left(\gamma_{i j}\right)_{i, j=1}^{N}$ with

$$
\gamma_{i j}=\left(1-\rho_{i j}\right) \sigma_{i} \sigma_{j}
$$

